INPLASY

PROTOCOL

To cite: Dong et al. The efficacy of Qigong exercises for post-stroke mental disorders and sleep disorders: protocol for a systematic review and meta-analysis. Inplasy protocol 202070051. doi:

10.37766/inplasy2020.7.0051

Received: 12 July 2020

Published: 14 July 2020

Corresponding author:

Xing Dong

dxtcm20@yeah.net

Author Affiliation:

Chengdu University of TCM

Support: Sichuan Science and technology

Review Stage at time of this submission: The review has not yet started.

Conflicts of interest:

The authors declare that they have no competing interests.

\section{The efficacy of Qigong exercises for post-stroke mental disorders and sleep disorders: protocol for a systematic review and meta-analysis}

Dong, X13 Zhang, RZ2; Guo, Y3; Chen, LF4; Liu, Y5.

Review question / Objective: Are Qigong exercises effective in improving mental and sleep outcomes in patients with poststroke? Which kind of Qigong have the most beneficial effects in post-stroke?

Condition being studied: Qigong has a long history in china, which has both the function of health and cure, especially in mental illness. Consequently, there are a huge number of studies reports the effectiveness of Qigong for post-stroke mental disorders and sleep disorders (PSMDs), but strongly conclusions have not been achieved owing to a wide variation in scopes, qualities, and outcome.

INPLASY registration number: This protocol was registered with the International Platform of Registered Systematic Review and Meta-Analysis Protocols (INPLASY) on 14 July 2020 and was last updated on 14 July 2020 (registration number INPLASY202070051).

\section{INTRODUCTION}

Review question / Objective: Are Qigong exercises effective in improving mental and sleep outcomes in patients with poststroke? Which kind of Qigong have the most beneficial effects in post-stroke?
Condition being studied: Qigong has a long history in china, which has both the function of health and cure, especially in mental illness. Consequently, there are a huge number of studies reports the effectiveness of Qigong for post-stroke mental disorders and sleep disorders 
(PSMDs), but strongly conclusions have not been achieved owing to a wide variation in scopes, qualities, and outcome.

\section{METHODS}

Search strategy: A comprehensive search of prominent medical and health science electronic databases: PubMed, Cochrane Library, Embase, China National Knowledge Infrastructure Database (CNKI), WANGFANG DATA, Chinese Biomedical Literature Database (CBM) was undertake till 30th June 2020 for Qigong for PSMDs without any language restriction.

Participant or population: This review includes post-stroke depression patients, no matter what reason caused stroke.

Intervention: Qigong-related exercise was the main intervention (e.g. Qigong, Baduanjin, Yijinjing, Wuqinxi). The duration and frequency of therapy are not limited.

Comparator: There is no exclusion based on comparator method for this review, and the patients could be treated with any type of control group including exercise, stretching, sham Qigong, waiting list control, or other treatments.

Study designs to be included: Randomized controlled trials with no limitations on blinding or publication types will be included.

Eligibility criteria: Randomized controlled trials (RCTs) with no limitations on binding published in English and Chinese up to June 30, 2020 will be included. Patients with primary diseases related PSMDs symptoms will be include, regardless of the underlying diseases, age, gender, education, ethnicity, and occupation. There will be no restrictions regarding language or publication status. Non-randomized trials, case reports, observational studies, and reviews will be excluded.

Information sources: The following database will be searched: PubMed, Web of Science, Embase, Cochrane Library, China Biology Medicine disc, China National
Knowledge Infrastructure (CNKI), WANGFANG, and VIP Data Knowledge Service Platform. Clinical trial registries are part of other resource searches, like ClinicalTrials.gov will be searched for ongoing trials with unpublished data.

Main outcome(s): The main outcome includes Hamilton depression scale, Hamilton anxiety scale, the mental health part of the MOS item short from health survey (SF-36), Generic Quality of Life Inventory-74, Center for Epidemiologic Studies Depression Scale, Pittsburgh sleep quality index.

Additional outcome(s): The additional outcomes include the effective rate and adverse events.

Data management: We use Noteexpress 3.2.0 software to read the titles and abstracts for a preliminary screening. When the titles and abstracts couldn't be definitively excluded, we downloaded the full text and filtered it again until all RCTs are confirmed.

Quality assessment / Risk of bias analysis: Two researchers assess the methodological quality of the included reviews according to Cochrane handbook 5.1.0, show an overall rating as high, unclear, low risk. In addition, we assess the evidence quality of concerning outcomes according to GRADE system. We classify evidence quality as high, moderate, low, or very low.

Strategy of data synthesis: The Revman $\mathbf{5 . 3}$ software is used to perform all statistical analyses. All outcomes are presented as continuous variables in this review. We will perform a pairwise meta-analysis using a random-effect model. To determine the effect size, risk rations with $95 \%$ confidence intervals will be calculated for dichotomous outcomes and the standard mean difference with $95 \%$ confidence intervals will be calculated for continuous outcomes. Depending on the heterogeneity assessed by the 12 statistic, a fixed- or random- effect model will be used. If there is statistical heterogeneity, sensitivity to 
explore the source of heterogeneity. We will then perform a Bayesian network metaanalysis using a random-effects model to analyze pooled data for different interventions.

Subgroup analysis: We apply a sensitivity analysis by combining the same models to various results to identify the source of heterogeneity and reliability of the various results.

Sensibility analysis: If there are adequate data available to analyze, we will conduct a sensitivity analysis on the primary outcomes to test the strength of the review conclusions, including the quality of the methods and studies, and the impact of sample size and missing data.

Language: English or Chinese-language.

Country(ies) involved: China.

Keywords: Qigong, post-stroke, mental disorder; sleep disorder; protocol; systematic analysis; meta-analysis.

Contributions of each author:

Author 1 - Xing Dong - was in charge of conceptualization and investigation, drafted the manuscript.

Author 2 - Renyan Zhang - provided statistical expertise.

Author 3 - Ying Guo - contributed to the development of the selection criteria, and the risk of bias assessment strategy.

Author 4 - Longfang Chen - contributed to the development of the selection criteria, and the risk of bias assessment strategy.

Author 5 - Yuan Liu - read, provided feedback and approved the final manuscript. 